\title{
Serum levels of Sex Hormone Binding Globulin (SHBG) are not predictive of prostate cancer diagnosis and aggressiveness: results from an italian biopsy cohort
}

\author{
Cosimo De Nunzio, Riccardo Lombardo, Simone Albisinni, Mauro Gacci, Andrea Tubaro \\ Department of Urology, Ospedale Sant'Andrea, University La Sapienza (CDN, RL, SA, AT), Rome, and \\ Department of Urology, Careggi Hospital (MG), University of Florence, Florence, Italy
}

\section{ABSTRACT}

Purpose: To explore the association between serum levels of Sex Hormone Binding Globulin (SHBG) and the risk of developing prostate cancer ( $\mathrm{PCa}$ ) as well as high grade disease in men undergoing prostate biopsy.

Materials and Methods: Between 2006 and 2012, we prospectively enrolled 740 patients with no history of PCa undergoing prostate biopsy. Before biopsy general data of the patient DRE, PSA and BMI were recorded. The risk of detecting cancer and high grade cancer was assessed as a function of SHBG using crude and adjusted logistic regressions. Results: Serum levels of SHBG were not associated with an increased risk of PCa or high grade disease. Age (OR 1.027 95\% CI 1.003-1.052 p = 0.027), DRE (OR 3.391 95\% CI 2.258-5.092 $\mathrm{p}=0.000$ ) and PSA (OR 1.078 95\% CI 1.037-1.120 p=0.000) were found to be independent predictors of prostate cancer risk. Age (OR 1.051 95\% CI 1.009-1.095 p = 0.016), DRE (OR 2.519 95\% CI 1.384-4.584 p = 0.000), BMI (OR 1.098 95\% CI 1.011-1.193 $\mathrm{p}=0.027$ ) and PSA (OR 1.074 95\% CI 1.014-1.137 $\mathrm{p}=0.015)$ were found to be independent predictors of high grade disease.

Conclusions: In our cohort of patients, serum levels of SHBG are not predictive of PCa or high grade disease. According to our experience SHBG should not be considered a biomarker in PCa diagnosis neither a marker for high grade disease.

\section{ARTICLE INFO}

\author{
Key words: \\ Prostatic Neoplasms; Biopsy; \\ Pathology; Sex Hormone- \\ Binding Globulin; Androgens
}

Int Braz J Urol. 2013; 39: 793-9

Submitted for publication:

May 17, 2013

Accepted after revision:

August 15, 2013

\section{INTRODUCTION}

Prostate cancer $(\mathrm{PCa})$ is a major health concern worldwide, being the second most common neoplasm and sixth cause of cancer-related death in the entire world (1). PSA is considered the standard marker to identify patients at risk of PCa. However, specially in men with low PSA values, several markers have been investigated in order to identify patients at risk of high grade, poorly differentiated PCa $(2,3)$.
The prostate is an androgen-dependent gland, requiring testosterone for its growth, differentiation and function (3-5). Androgens have been well investigated in the development and progression of PCa but their exact role hasn't been elucidated yet. Moreover SHBG is well known to regulate levels of sex hormones and therefore might influence PCa genesis and progression directly or indirectly. Recent studies have evaluated the relationship between serum levels of dihydrotestosterone, testosterone, free testosterone, estrogens, SHBG and the development and progression 
of PCa (3,6-9). The role of these hormones in the pathogenesis of PCa is complex and no real consensus has been reached regarding their possible clinical implications.

The use and identification of new PCa biomarkers would help urologists in decision making and developing normograms with the aim of better identifying patients at risk of $\mathrm{PCa}$ and particularly high grade disease (3). The aim of our study was to evaluate the relationship between levels of SHBG and PCa in a group of patients undergoing transrectal prostate biopsies in order to identify a possible relationship between SHBG serum levels and PCa diagnosis and aggressiveness.

\section{MATERIALS AND METHODS}

After an Internal Review Board approval, between 2006 and 2012, a consecutive series of patients with no known history of PCa were referred to our department to undergo initial prostate biopsy because of an abnormal finding on digital rectal examination (DRE) and/or an elevation of serum levels of PSA ( $>4 \mathrm{ng} / \mathrm{mL}$ ). Patients with uncontrolled diabetes, thyroid disease, hyperprolactinemia, hypoalbuminemia or liver disease were excluded from the cohort. DRE was performed by a senior staff urologist and judged positive if suggestive of cancer. Body mass index (BMI) was calculated as $\mathrm{kg} / \mathrm{m}^{2}$. Every patient underwent $12-$ core TRUS (Transrectal Ultrasound) biopsy with the same scheme, following our department's protocol (10). All biopsies were performed and pathologically reviewed in the same institute. Low grade disease was defined as Gleason $\leq 6$ and high grade disease as Gleason $\geq 7$.

On the day of biopsy, after obtaining a dedicated informed consent, blood specimens were drawn before the procedure at 8:00 AM (all men were fasting from the night before). PSA, SHBG and testosterone were measured. Blood samples were kept at $4^{\circ}$ until serum was separated by centrifugation. Serum and plasma aliquots were then stored at $-80^{\circ}$ until assay. The IMMULITE 2000 (Siemens, Germany) chemoluminescent enzyme immunoassay method which is a solid phase, sequential chemiluminescent immunometric assay was used to determine PSA $(\mathrm{ng} / \mathrm{mL})$, SHBG $(\mathrm{pg} /$
$\mathrm{mL}$ ) and testosterone $(\mathrm{ng} / \mathrm{mL})$ concentrations. However, testosterone serum levels were not evaluated in this study as they have been previously investigated by our group (9).

\section{Statistical analysis}

Statistical analysis was performed using SPSS software. Evaluation of data distribution confirmed a not normal distribution of the study dataset. Differences between groups of patients in medians for quantitative variables and differences in distributions for categorical variables were tested with the Kruskal Wallis one way analysis of variance and Chi-square test, respectively. Using multiple logistic regression with the enter method, the statistical significant variables as assessed in univariate analysis were entered and investigated as predictors of prostate cancer presence vs. absence and in a separate model comparing high grade vs. low-grade among men with cancer at biopsy. The logistic regression analysis was carried out using date from patients whose complete data were available. The variables considered for entry into the model included Age, PSA, BMI, DRE and SHBG. Receiver operator characteristic curves (ROC) were produced to evaluate the area under the curve (AUC) and the diagnostic performance of PSA, SHBG and BMI for the detection of PCa and high grade PCa. An alpha value of 5\% was considered as the threshold for significance. Data are presented as median with Inter Quartile Range (IQR) and mean \pm standard deviation (SD). Odds ratios and 95\% Confidence Interval (CI) were calculated for the parameters in each group using PCa negative and Gleason 6 as reference group.

\section{RESULTS}

Overall, 740 patients were prospectively enrolled, median age was 68 (IQR: 62/73) years, median BMI was 27.1 (IQR:25.0/29.4) kg/m²; median PSA was 6.0 (IQR: 4.3/8.8) ng/mL, median SHBG level was 38.4 (IQR: 29.7/49.4) pg/mL (Table-1). 150/740 (20\%) patients presented a positive DRE. Overall 275/740 (37.2\%) patients presented PCa on biopsy and out of them 120/275 $(44 \%)$ presented high grade PCa (Gleason score $\geq$ 
Table 1 - Baseline characteristics of the cohort population.

\begin{tabular}{lcc}
\hline & Mean \pm SD & Median (IQR) \\
\hline Age (years) & $67.0 \pm 7.94$ & $68(62 / 73)$ \\
BMI $\left(\mathrm{kg} / \mathrm{m}^{2}\right)$ & $27.45 \pm 3.79$ & $27.1(25.0 / 29.4)$ \\
PSA $(\mathrm{ng} / \mathrm{mL})$ & $7.2 \pm 4.8$ & $6.0(4.3 / 8.8)$ \\
SHBG $(\mathrm{pg} / \mathrm{mL})$ & $41.4 \pm 17.4$ & $38.4(29.7 / 49.4)$ \\
\hline
\end{tabular}

7). More precisely 157/275 (57\%) had a Gleason 6, $73 / 275(27 \%)$ a Gleason 7, 23/275 (8\%), a Gleason $8,18 / 275$ (7\%), a Gleason 9 and 4/270 (1\%) a Gleason 10 disease.

Patients with PCa were significantly older $(p=0.000)$, presented a higher PSA $(p=0.000)$ when compared to patients with a negative biopsy. No significant differences were observed in terms of BMI and SHBG levels (Table-2). On the multivariate analysis Age, PSA and DRE were found to be independent predictors of prostate cancer risk (Table-3). Risk of having prostate cancer is increased by $2.7 \%$ per unit of age, by 239\% if DRE was positive and by $7.8 \%$ per unit of PSA.

Patients with high grade disease were significantly older $(p=0.000)$, presented a high- er PSA $(p=0.035)$ and higher BMI $(p=0.050)$ while no difference was found in terms of SHBG serum levels (Table-2). On multivariate analysis Age, DRE, BMI and PSA were found to be independent predictors of high grade disease (Table-3). Risk of high grade disease is increased $5.1 \%$ per unit of age, by $152 \%$ if DRE, by $9.8 \%$ per unit of BMI and by $7.8 \%$ per unit of PSA. In addition ROC analyses were applied to evaluate SHBG, BMI and the presence of cancer (Figure-1). SHBG produced an area under the curve (AUC $=0.517 ;$ CI: $0.474-0.561 ; p=0.428)$ that did not seem to qualify to predict PCa. BMI produced an area under the curve that did not seem to qualify to predict PCa (AUC $=0.503 ; \mathrm{CI}$ : $0.459-0.547 ; \mathrm{p}=0.882$ ). PSA served as a control (AUC $=0.595 ; \mathrm{CI}: 0.553-0.638 ; \mathrm{p}=0.000$ ). Furthermore, ROC analyses were applied to evaluate $17 \mathrm{BE}$ levels, BMI and the presence of high grade PCa (Figure-1). SHBG produced an area under the curve (AUC $=0.524 ; \mathrm{CI}: 0.453-0.596$; $\mathrm{p}=0.596$ ) that did not seem to qualify to predict high grade $\mathrm{PCa}$. BMI produced an area under the curve (AUC $=0.569 ;$ CI: 0.500-0.639; $p=0.05$ ) that qualified to predict high grade disease. PSA served as a control (AUC $=0.575$; CI: 0.505$0.645 ; p=0.035$ ).

Table 2 - Clinical and pathological characteristics of the cohort.

\begin{tabular}{|c|c|c|c|c|c|c|c|}
\hline & & No cancer & Cancer & $\mathrm{p}$ & $\begin{array}{c}\text { Gleason } \\
\text { Score } \leq 6\end{array}$ & $\begin{array}{c}\text { Gleason } \\
\text { Score } \geq 7\end{array}$ & $p$ \\
\hline Patients & & $465 / 740(62.8 \%)$ & $275 / 740(37.2 \%)$ & & $155 / 275(56 \%)$ & $120 / 275(44 \%)$ & \\
\hline $\mathrm{DRE}+$ & & $58 / 465(12 \%)$ & $92 / 275(33 \%)$ & 0.000 & $35 / 155(23 \%)$ & $57 / 120(48 \%)$ & 0.000 \\
\hline \multirow{2}{*}{ Age (years) } & Mean \pm SD & $66.02 \pm 7.9$ & $68.9 \pm 7.6$ & \multirow{2}{*}{0.000} & $67.37 \pm 7.72$ & $70.91 \pm 7.1$ & \multirow{2}{*}{0.000} \\
\hline & Median (IQR) & $67(60 / 72)$ & $70(64 / 74)$ & & $68(61 / 73)$ & $72(66 / 76)$ & \\
\hline \multirow{2}{*}{$\mathrm{BMI}\left(\mathrm{Kg} / \mathrm{m}^{2}\right)$} & Mean \pm SD & $27.45 \pm 3.6$ & $27.45 \pm 4.02$ & \multirow{2}{*}{0.881} & $27.02 \pm 3.62$ & $28.00 \pm 4.44$ & \multirow{2}{*}{0.050} \\
\hline & Median (IQR) & $27.0(25.0 / 29.4)$ & $27.2(24.8 / 29.6)$ & & $26.8(24.8 / 29.3)$ & $27.7(25.2 / 30.5)$ & \\
\hline \multirow{2}{*}{ PSA (ng/mL) } & Mean \pm SD & $6.53 \pm 4.0$ & $8.4 \pm 5.6$ & \multirow{2}{*}{0.000} & $7.5 \pm 4.61$ & $9.6 \pm 6.7$ & \multirow{2}{*}{0.035} \\
\hline & Median (IQR) & $5.6(4.0 / 8.0)$ & $6.7(4.6 / 10.9)$ & & $6.2(4.5 / 9.6)$ & $7.2(4.8 / 12.3)$ & \\
\hline \multirow{2}{*}{ SHBG $(p g / m L)$} & Mean \pm SD & $40.7 \pm 16.2$ & $42.7 \pm 19.3$ & \multirow{2}{*}{0.428} & $41.1 \pm 16.5$ & $24.7 \pm 22.5$ & \multirow{2}{*}{0.492} \\
\hline & Median (IQR) & $38.1(29.4 / 49.0)$ & $39.2(30.1 / 49.9)$ & & $39.4(30.3 / 48.3)$ & $38.8(29.5 / 53.5)$ & \\
\hline
\end{tabular}


Table 3 - Exploring the association of SHBG and prostate cancer risk.

\begin{tabular}{lcccccc}
\hline & & Overall Cancer Risk & \multicolumn{3}{c}{ Risk of having High grade disease } \\
\hline Age & OR & $95 \% \mathrm{Cl}$ & $\mathrm{P}$ & $\mathrm{OR}$ & $95 \% \mathrm{Cl}$ & $\mathrm{P}$ \\
DRE & 1.027 & $1.003-1.052$ & 0.027 & 1.051 & $1.009-1.095$ & 0.016 \\
BMI & 3.391 & $2.258-5.092$ & 0.000 & 2.519 & $1.384-4.584$ & 0.000 \\
PSA & 0.988 & $0.941-1.037$ & 0.627 & 1.098 & $1.011-1.193$ & 0.027 \\
SHBG & 1.078 & $1.037-1.120$ & 0.000 & 1.074 & $1.014-1.137$ & 0.015 \\
\hline
\end{tabular}

Figure 1 - Receiver operating characteristic analysis curve comparing SHBG serum levels and BMI in patients with and without prostate cancer (ROC Curve 1) and in patients with low vs high grade disease (ROC Curve 2).

ROC Curve 1

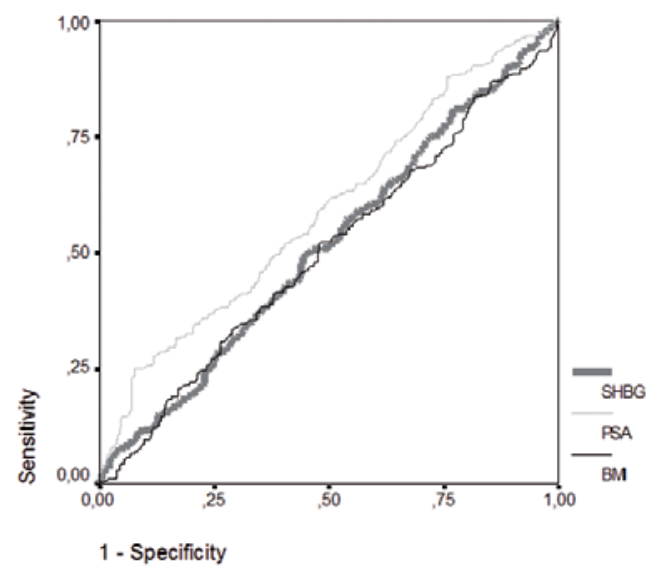

\section{DISCUSSION}

Our experience confirmed how older patients with higher PSA are at increased risk of PCa diagnosis. Moreover higher PSA and higher BMI in patients scheduled for prostate biopsy have an increased risk of developing high grade disease as showed by our previous studies (11). Clearly the strong association between DRE and prostate cancer risk at biopsy in our series confirms the internal validity of our results and it is in line with the peer review literature $(3,12)$.
ROC $\cdot$ Curve 2

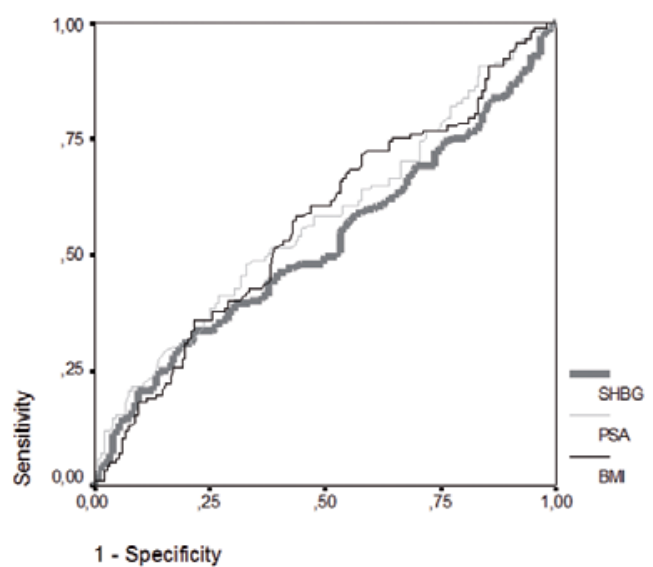

In our series obesity is a prevalent condition and it is associated with an increased risk of high grade disease in patients with PCa. This data confirmed our previous experiences where we observed how obesity is a significant independent risk factor for high grade Gleason score when PCa is diagnosed by prostate biopsy $(3,9,13)$. Furthermore, the same observations were reported in other studies evaluating the possible relationship between obesity and PCa development and progression $(14,15)$. Although further studies are needed to confirm these findings and to evaluate which 
biological factors are responsible for the observed results, our experience add further evidence on the relationship between obesity and PCa.

SHBG is a serum globulin that binds testosterone in plasma regulating though its free component. SHBG has been associated with PCa genesis (16-18). Berndt et al. (16) found a strong relationship between SHBG gene polymorphism and risk of developing PCa. Moreover SHBG receptors have been found on prostate cells and their activation leads to an increase in cAMP possibly activating oncological pathways $(17,18)$.

However, in our experience, no significant difference in SHBG serum levels was found between patients with or without PCa. Specifically SHBG serum levels were not associated with an increased risk PCa diagnosis on biopsy or with an increased risk of high grade disease in patients with PCa. Moreover the ROC analysis showed how SHBG predicts PCa or high PCa no more accurately than a coin toss. Although our experience is preliminar to obtain a definitive conclusion, it is the largest cohort study available regarding the association between SHBG and PCa diagnosis in patients with elevated PSA and/or abnormal DRE scheduled for prostate biopsy. Most of the available studies have evaluated the role of SHBG exclusively in patients scheduled for radical retropubic prostatectomy (RRP). Lee et al. found that SHBG preoperative levels are predictive of extraprostatic extension of tumour, SHBG serum levels achieved the role of independent predictor of extraprostatic extension on multivariate analysis (OR $=1.342, p=0.039)$ (19). Salonia et al. in the same settings found that SHBG levels were independent predictors of extracapsular extension in men undergoing radical prostatectomy $(\mathrm{OR}=$ $1.01, \mathrm{p}<0.001)$ (20). Moreover high SHBG levels increase the risk of nodal metastases by $11 \%$ per unit $(\mathrm{OR}=1.11, \mathrm{p}<0.001)$ and the addition of SHBG increases the predictive accuracy of the base model using clinically established predictors from $72.7 \%$ to $82.8 \%$ (21). However as observed in our experience they found no correlation between SHBG levels and high grade disease $(\mathrm{p}=$ 0.61) (22). Although all these studies differ from our series, a complete comparison is not possible as we evaluated the serum levels of SHBG in a population of patients at high risk of prostate cancer while most of the available evidence considered patients scheduled for RRP.

Case-control studies have also evaluated the relationship between hormonal status and PCa diagnosis. Sawada et al. analysed plasma testosterone and SHBG and the risk of PCa among Japanese men and found that neither testosterone neither SHBG levels were associated with an increased risk for total PCa (respectively: $p=0.49 ; p=0.89$ ) (18). In the same way Heikkila et al. in their case control study found no correlation between SHBG levels and PCa risk (23). On the other hand, Gann et al. made a prospective study of sex hormone levels and risk of $\mathrm{PCa}$ and found an inverse trend of risk for PCa with increasing levels of SHBG $(p=0.07)(24)$. Finally, a metanalysis from Roddam et al., considering 18 prospective studies on sex hormones and PCa found no correlation between serum concentrations of sex hormones and PCa risk and a modest inverse association between SHBG levels and PCa risk with a relative risk reduction of 14\% (25). It is important to point out that one of the possible limitations in comparing these studies is the time of venous sampling. SHBG levels are quite stable during the day although variations in SHBG levels in a longer period of time hasn't been evaluated yet (26). Moreover serum levels of SHBG are influentiated by IGF-1 levels and BMI (26). Data analysed by the available studies differ in these terms and so far are difficult to compare. From all these studies it is evident that the exact role of SHBG in PCa genesis and progression is still an open debate and more standardised and multicentric studies are needed to clarify these preliminary observations.

It is correct to point out the limitation of our study. Cancer aggressiveness was classified according to biopsy specimens and TRUS-guided prostate biopsy is associated with a certain degree of under and overestimating of PCa, however with a single centre study and a single uropathologist reviewing all biopsies this possible bias was reduced. Another limitation of our study is that a single assessment of circulating SHBG concentrations may not be reliable clinically; however, as reported by Salonia et al., a single fasting morning venous blood sample determines the assessment of the hormone status more user friendly in every day clinical practice 
(20). However, at present our study is the largest prospective series evaluating the association between SHBG levels and PCa risk among men with elevated PSA or/and abnormal digital rectal examination scheduled for prostate biopsy and using the same biopsy template for the entire cohort.

We have also considered the possibility of running a separate analysis for patients with Gleason 8-10 (excluding patients with Gleason 7) but for the sake of homogeneity with papers published in the peer review literature $(7,9,10,11,13,21)$, we decided to run a dichotomous analysis grouping in the high risk population also men with a Gleason score 7. Furthermore, our approach to define high grade prostate cancer by Gleason score $\geq 7$ is also in line with a number of published papers $(27,28)$. However, we acknowledge that further research and large number of patients are needed to explore potential differences in the different Gleason score subgroups.

\section{CONCLUSIONS}

In our cohort of patients at risk of $\mathrm{PCa}$, the serum level of SHBG is not a significant predictor of prostate cancer diagnosis and aggressiveness on initial prostate biopsy. According to our experience, SHBG, should not be considered a reliable marker to predict poorly differentiated cancer in the setting of initial prostate biopsy. Further studies are needed to confirm our findings in other populations and to evaluate which biological factors related to serum SHBG levels are involved to prostate cancer development and progression.

\section{ABBREVIATIONS}

$\mathrm{PCa}=$ Prostate Cancer

SHBG = Sex Hormone Binding Globulin

DRE $=$ Digital Rectal Examination

$\mathrm{BMI}=$ Body Mass index

ROC $=$ Receiver Operator Characteristics

AUC $=$ Area Under the Curve

$\mathrm{IQR}=$ Inter Quartile Range

$\mathrm{CI}=$ Confidence interval

RRP = Radical retropubic prostatectomy

TRUS $=$ Transrectal Ultrasound

\section{CONFLICT OF INTEREST}

\author{
None declared.
}

\section{REFERENCES}

1. Center MM, Jemal A, Lortet-Tieulent J, Ward E, Ferlay J, Brawley 0 , et al.: International variation in prostate cancer incidence and mortality rates. Eur Urol. 2012; 61: 1079-92.

2. Castelli T, Cimino S, Magno C, Morgia G: Molecular markers for prostatic cancer. Front Biosci (Elite Ed). 2010; 2: 641-56.

3. De Nunzio C, Lombardo R, Leonardo C, Franco G, Gacci $M$, Presicce $F$, et al.: Serum levels of $17-\beta$-estradiol are not predictive of prostate cancer diagnosis and aggressiveness: Results from an Italian biopsy cohort. Urol Oncol. 2013. In Press

4. De Nunzio C, Miano R, Trucchi A, Finazzi Agrò E, Tubaro $A$ : Finasteride for prostatic disease: an updated and comprehensive review. Expert Opin Drug Metab Toxicol. 2008; 4: $1561-8$.

5. Huggins G, Hodge GB: Studies on prostatic cancer. I. The effect of castration, of estrogen and of androgen injection on serum phosphatase in metastatic carcinoma of the prostate. Cancer Res. 1941; 1: 293-7.

6. Wirén S, Stocks T, Rinaldi S, Hallmans G, Bergh A, Stenman UH, et al.: Androgens and prostate cancer risk: a prospective study. Prostate. 2007; 67: 1230-7.

7. Schatzl G, Madersbacher S, Thurridl T, Waldmüller J, Kramer G, Haitel A, et al.: High-grade prostate cancer is associated with low serum testosterone levels. Prostate. 2001; 47: 52-8.

8. Hoffman MA, DeWolf WC, Morgentaler A: Is low serum free testosterone a marker for high grade prostate cancer? J Urol. 2000; 163: 824-7.

9. Albisinni S, De Nunzio C, Tubaro A, Barry WT, Banez LL, Freedland SJ: Greater percent-free testosterone is associated with high-grade prostate cancer in men undergoing prostate biopsy. Urology. 2012; 80: 162-7.

10. De Nunzio C, Trucchi A, Miano R, Stoppacciaro A, Fattahi $\mathrm{H}$, Cicione $A$, et al.: The number of cores positive for high grade prostatic intraepithelial neoplasia on initial biopsy is associated with prostate cancer on second biopsy. J Urol. 2009; 181: 1069-74; discussion 1074-5.

11. De Nunzio C, Freedland SJ, Miano R, Trucchi A, Cantiani A, Carluccini A, et al.: Metabolic syndrome is associated with high grade gleason score when prostate cancer is diagnosed on biopsy. Prostate. 2011. In Press 
12. Wolf AM, Wender RC, Etzioni RB, Thompson IM, D'Amico AV, Volk RJ, et al.: American Cancer Society guideline for the early detection of prostate cancer: update 2010. CA Cancer J Clin. 2010; 60: 70-98.

13. De Nunzio C, Freedland SJ, Miano L, Finazzi Agrò E, Bañez L, Tubaro A: The uncertain relationship between obesity and prostate cancer: an Italian biopsy cohort analysis. Eur J Surg Oncol. 2011; 37: 1025-9.

14. Allott EH, Masko EM, Freedland SJ: Obesity and prostate cancer: weighing the evidence. Eur Urol. 2013; 63: 800-9.

15. Freedland SJ, Wen J, Wuerstle M, Shah A, Lai D, Moalej B, et al.: Obesity is a significant risk factor for prostate cancer at the time of biopsy. Urology. 2008; 72: 1102-5.

16. Berndt SI, Chatterjee N, Huang WY, Chanock SJ, Welch R, Crawford ED, et al.: Variant in sex hormone-binding globulin gene and the risk of prostate cancer. Cancer Epidemiol Biomarkers Prev. 2007; 16: 165-8.

17. Weigel NL, Zhang Y: Ligand-independent activation of steroid hormone receptors. J Mol Med (Berl). 1998; 76: 469-79.

18. Sawada N, Iwasaki M, Inoue M, Sasazuki S, Yamaji T, Shimazu T, et al.: Plasma testosterone and sex hormonebinding globulin concentrations and the risk of prostate cancer among Japanese men: a nested case-control study. Cancer Sci. 2010; 101: 2652-7.

19. Lee SE, Chung JS, Han BK, Park CS, Moon KH, Byun SS, et al.: Preoperative serum sex hormone-binding globulin as a predictive marker for extraprostatic extension of tumor in patients with clinically localized prostate cancer. Eur Urol. 2008; 54: 1324-32.

20. Salonia A, Gallina A, Briganti A, Zanni G, Suardi N, Capitanio $\mathrm{U}$, et al.: Sex hormone-binding globulin is a significant predictor of extracapsular extension in men undergoing radical prostatectomy. BJU Int. 2011; 107: 1243-9.

21. Salonia $A$, Briganti $A$, Gallina $A$, Karakiewicz $P$, Shariat $S$, Freschi M, et al.: Sex hormone-binding globulin: a novel marker for nodal metastases prediction in prostate cancer patients undergoing extended pelvic lymph node dissection. Urology. 2009; 73: 850-5.
22. Salonia A, Gallina A, Briganti A, Suardi N, Capitanio U, Abdollah $\mathrm{F}$, et al.: Circulating estradiol, but not testosterone, is a significant predictor of high-grade prostate cancer in patients undergoing radical prostatectomy. Cancer. 2011; 117: 5029-38.

23. Heikkilä R, Aho K, Heliövaara M, Hakama M, Marniemi J, Reunanen A, et al.: Serum testosterone and sex hormonebinding globulin concentrations and the risk of prostate carcinoma: a longitudinal study. Cancer. 1999; 86: 312-5.

24. Gann PH, Hennekens CH, Ma J, Longcope C, Stampfer MJ: Prospective study of sex hormone levels and risk of prostate cancer. J Natl Cancer Inst. 1996 ; 88: 1118-26.

25. Roddam AW, Allen NE, Appleby P, Key TJ, Endogenous Hormones and Prostate Cancer Collaborative Group: Endogenous sex hormones and prostate cancer: a collaborative analysis of 18 prospective studies. J Natl Cancer Inst. 2008; 100: 170-83.

26. Pasquali R, Casimirri F, Cantobelli S, Melchionda N, Morselli Labate AM, Fabbri R, et al.: Effect of obesity and body fat distribution on sex hormones and insulin in men. Metabolism. 1991; 40: 101-4.

27. Thompson IM, Goodman PJ, Tangen CM, Lucia MS, Miller GJ, Ford LG, et al.: The influence of finasteride on the development of prostate cancer. N Engl J Med. 2003; 349: 215-24.

28. Andriole GL, Bostwick DG, Brawley OW, Gomella LG, Marberger M, Montorsi F, et al.: Effect of dutasteride on the risk of prostate cancer. N Engl J Med. 2010; 362: 1192-202.

\footnotetext{
Correspondence address:

Cosimo De Nunzio, MD, PhD Ospedale Sant'Andrea University La Sapienza Roma, Italy

Telephone: + 3906 3377-7716 E-mail: cosimodenunzio@virgilio.it
} 